\title{
Embedded Technology for vehicle cabin safety Monitoring and Alerting System
}

\author{
V.Ramya ${ }^{1}$, B. Palaniappan ${ }^{2}$ \\ ${ }^{1}$ Assistant Professor, Department of CSE, Annamalai University, Chidambaram, India. \\ Email:ramyshri@yahoo.com \\ ${ }^{2}$ Dean, FEAT, Head, Department of CSE, Annamalai University, Chidambaram, India. \\ ${ }^{2}$ Email: bpau2002@yahoo.co.in
}

\begin{abstract}
ABSRACT
Motor vehicles are the prime source of transportation where vehicles with A/C play a major part. This paper designs an embedded system for a vehicle cabin, which senses the gases like carbon-monoxide and oxygen and displayed at each and every second. If the level of the CO increases than the normal level (30ppm) or the level of the oxygen decreases than the normal level (19\%) then an alarm is generated automatically and also ventilation is provided immediately. A warning message is sent to the authorized user via GSM. The advantage of this system is proper detection and faster response time leading to faster diffusion of the situation, compared with the manual methods.
\end{abstract}

\section{KEYWORDS}

Atmel microcontroller; Embedded System; Gas detecting sensors; GSM Modem; Vehicle Safety;

\section{INTRODUCTION}

Motor vehicles are they not only the prime source of transportation, but also the prime source of pollution. These motor vehicles contribute to over $30 \%$ of the hazardous gases that are released into the atmosphere. Outdoor pollution levels are the key concern, but the quality of air inside the vehicle plays a major part. As the vehicle cabin is small area, any suspended particulate such as dust, fumes, smokes entering in to it through either ventilation or through windows can cause serious health problems to the person inside. These particulate matters can cause problems which may differ from acute and chronic effects on human health. The health effects range from minor irritation of eyes and the upper respiratory system to chronic respiratory disease, heart disease, lung cancer and even death .

Even if the vehicle is equipped with the air cabin filters that are present inside the cabin so as to reduce the effect of the unwanted particulate, they cannot be a viable solution to control it. Even though the producers of various vehicles mainly concentrate on these safety precautions, the carelessness of the end users or the drivers regarding the condition of the vehicle may cause serious damage not only to the vehicles but also to the human lives. Such that even the minor eye irritation can make the driver to lose his concentration on the road, which may lead to serious accidents. Hence to overcome these types of problems there is a need to monitor several gases

DOI : 10.5121/ijcsea.2012.2207 
such as carbon monoxide (CO) and the oxygen level is required to ensure complete vehicle occupant comfort and safety. The embedded system is used inside a vehicle cabin such that the presence or leakage of toxic gases can be detected by the gas sensors and proper precautions can be taken to avoid the driver from getting fatigue or drowsiness [8].

Headache, dizziness, weakness, nausea, vomiting, chest pain, and confusion are the most common symptoms of $\mathrm{CO}$ poisoning. This paper designs an embedded system for toxic gas $\mathrm{CO}$ and oxygen level detection inside the vehicle cabin and to develop a sensing system using a sensor array and microcontroller. If the toxic gas reaches the maximum threshold level, the detection unit detects, an alarm is generated immediately and the ventilation will be provided automatically. And then an SMS is send to the authorized user through the GSM module [7].

\subsection{Related work}

In the year of 2008, LIU zhen-ya, WANG Zhen-dong and CHEN Rong, "Intelligent Residential Security Alarm and Remote Control System Based On Single Chip Computer", the paper focuses on, Intelligent residential burglar alarm, emergency alarm, fire alarm, toxic gas leakage remote automatic sound alarm and remote control system, which is based on 89c51 single chip computer. The system can be automatic alarm, automatic calling the police hotline number. It can be used voice alarm and show alarm occurred address. It can set up and modify user password. It can be recordable and voice suggestion. It can be used telephone remote control electrical power [8].

In the year of 2008, Chen Peijiang and Jiang Xuehhua, "Design and implementation of Remote Monitoring System Based on GSM", this paper focuses on the wireless monitoring system, because the wireless remote monitoring system has more and more application, a remote monitoring system based on SMS of GSM is presented. Based on the total design of the system, the hardware and software of the system is designed. In this system, GSM network is a medium for transmitting the remote signal. The system includes two part which are the monitoring centre and the remote monitoring station. The monitoring consists of a computer and a TC35 communication module of GSM. The computer and the TC35 are connected by RS232. The remote monitoring station includes a TC35 communication module of GSM, a MSP430F149 MCU, a display unit, various sensors, data gathering and processing unit. The software of the monitoring center and the remote monitoring station is designed by using VB. The result of demonstration shows that the system can monitor and control the remote communication between the monitoring center and the remote monitoring station, and the remote monitoring function is realized [7].

In the year of 2006, Ioan Lita, Ion Bogdan Cioc and Daniel Alexandru Visan, "A New Approach of Automatic Localization System Using GPS and GSM/GPRS Transmission", this paper focuses on, a low cost automotive localization system using GPS and GSM-SMS services. The system permits localization of the automobile and transmitting the position to the owner on his mobile phone as a short message (SMS) as his request. The system can be interconnected with the car alarm system and alert the owner, on his mobile phone, about the events that occurs with his car when it is parked. The system is composed by a GPS receiver, a microcontroller and a GSM phone. Additional, the system can be settled for acquiring and transmitting of information, when requested about automobile status and parameters or alert when it started engine, system can be used as navigation system. Optional, the system can be used as car low cost solution for automobile position localizing and status, very useful in case of watching and monitoring by their parents, as well as in car tracking system application. The proposed solution can be used in other types of application, where the information needed are requested rarely and at irregular period of time [16]. 
In the year of 2002, K. Galatsis, W. Wlodarsla, K. Kalantar-Zadeh and A. Trinchi, "Investigation of gas sensors for vehicle cabin air quality monitoring", this paper focuses on, car cabin air quality monitoring can be effectively analyzed using metal oxide semiconducting (MOS) gas sensors. In this paper, commercially available gas sensors are compared with fabricated Moo3 based sensors possessed comparable gas sensing properties. The sensor has response $74 \%$ higher relative to the hest commercial sensor tested [21]. In the year 2000, K. Galatsis, W. Woldarsla, Y.X. Li and K. Kalantar-zadeh, "A Vehicle air quality monitor using gas sensors for improved safety", this paper focuses on A vehicle cabin air quality monitor using carbon monoxide (CO) and oxygen (02) gas sensors has been designed, developed and on-road tested. The continuous monitoring of oxygen and carbon monoxide provides added vehicle safety as alarms could be set off when dangerous gas concentrations are reached, preventing driver fatigue, drowsiness, and exhaust gas suicides. CO concentrations of $30 \mathrm{ppm}$ and oxygen levels lower than $19.5 \%$ were experienced whilst driving [22].

\section{HARDWARE DESCRIPTION}

\subsection{Atmel 89c51 Microcontroller}

The AT89C51 is a low-power, high-performance CMOS 8-bit microcomputer with 4K bytes of Flash programmable and erasable read only memory (PEROM). The device is manufactured using Atmel's high-density nonvolatile memory technology and is compatible with the industrystandard MCS-51 instruction set and pin out. The on-chip Flash allows the program memory to be reprogrammed in-system or by a conventional Nonvolatile memory programmer. By combining a versatile 8-bit CPU with Flash on a monolithic chip, the Atmel AT89C51 is a powerful microcomputer which provides a highly-flexible and cost-effective solution to many embedded control applications. Figure1 shows the model of Atmel 89c51. In addition, the AT89C51 is designed with static logic for operation down to zero frequency and supports two software selectable power saving modes. The Idle Mode stops the CPU while allowing the RAM, timer/counters, serial port and interrupt system to continue their functioning. The Power-down Mode saves the RAM contents but freezes the oscillator, disabling all other chip functions until the next hardware reset. [17].

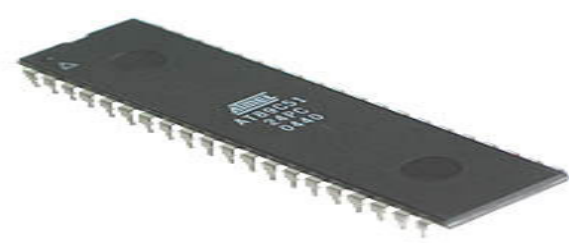

Figure 1. Atmel 89c51

\subsubsection{Features}

- Compatible with MCS-51 $1^{\mathrm{TM}}$ Products

- 4K Bytes of In-System Reprogrammable Flash Memory

Endurance: 1,000 Write/Erase Cycles

- Fully Static Operation: $0 \mathrm{~Hz}$ to $24 \mathrm{MHz}$

- Three-level Program Memory Lock

- 128 x 8-bit Internal RAM 
- 32 Programmable I/O Lines

- Two 16-bit Timer/Counters

- Six Interrupt Sources

- Programmable Serial Channel

- Low-power Idle and Power-down Modes

\subsection{Block Diagram}

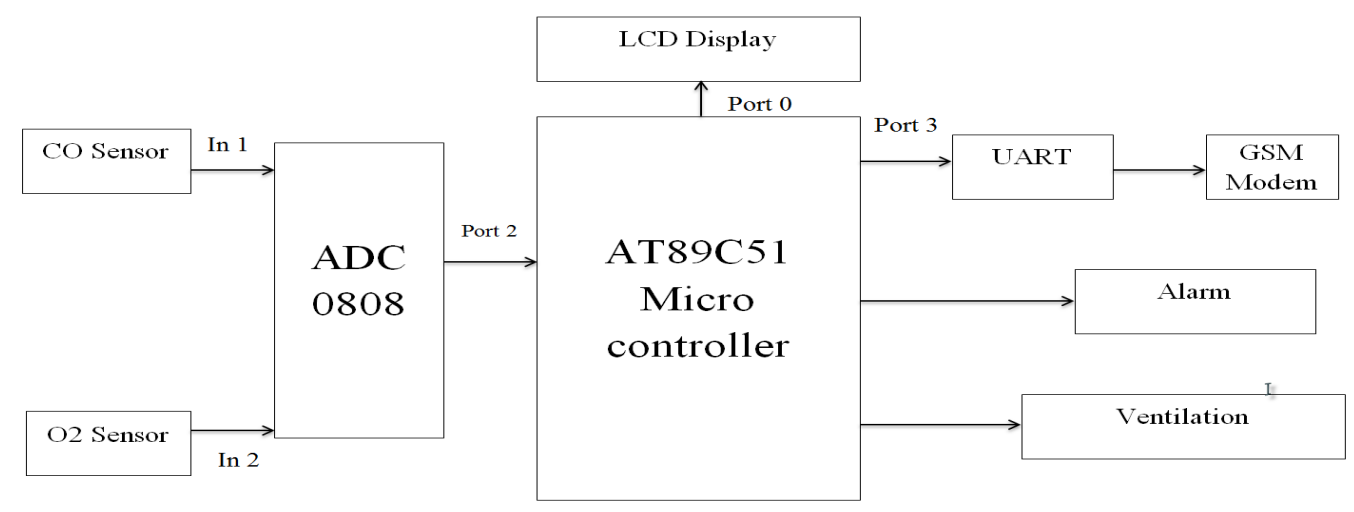

Figure 2. Block Diagram of the Proposed System

The AT89C51 micro controller is connected to the Analog to digital converter such that the input from the sensors are converted into digital input and then sent to the microcontroller it then displays the result through LCD. If a critical situation is experienced then an alarm is given for alerting the diver and ventilation is provided as an exhaust and as a remedy measure. A text message is sent to the authorized user indicating the critical situation of the vehicle. The block diagram of the proposed system is shown in figure2.

\subsection{Gas Sensors}

A gas sensor or detector is a device which detects the presence of various gases within the area, usually as the part of a safety system. This type of equipment is used to detect gas leakage and interface with the control system so as to shutdown the process easily. A gas detector can also sound an alarm in the area where the leak is occurring, giving them the opportunity to leave the area. This type of devices is important because there are many gases that can be harmful to organic life, such as humans and animals. Gas detectors are used to detect combustible, flammable and toxic gases, and also oxygen depletion. These types of devices are used widely in devices and can found in various locations. These are actually battery operated, and also they transmit warnings via a series of audible signals such as alarms and visible signals such as flashlights, as they meet dangerous levels. Originally detectors were produced to detect a single gas. But the modern units are capable of detecting several toxic gases or combustible gases or both [21]. These gas sensors can be classified according to the operation mechanism such as semiconductor, oxidation, catalytic, infrared, etc. But these can also be classified mainly into two types, they are,

- Portable Gas Sensors.

- Fixed Gas Sensors. 


\subsubsection{Health effects of Carbon Monoxide (CO)}

$\mathrm{CO}$ is a colorless, odorless and tasteless compound produced by incomplete combustion. It is often referred to as "Silent killer" because it is virtually undetectable without using detection technology. $\mathrm{CO}$ is found in combustion fumes produced by vehicles, gasoline engines, stoves and heating systems, people and animals in these $\mathrm{CO}$ enclosed spaces can be poisoned by breathing it. Even loss of consciousness and death can be caused due to high level inhalation of CO. CO poisoning can be difficult to diagnose and hence People who are sleeping or intoxicated can die from $\mathrm{CO}$ poisoning before ever experiencing symptoms. Elevated levels of $\mathrm{CO}$ can be dangerous to human depending on the amount present and length of exposure. Smaller concentration can be harmful over longer periods of time. People with chronic heart disease, anemia, or respiratory problems and unborn babies, infants are more susceptible to its effects. $\mathrm{CO}$ emissions and concentrations in urban and roadside air are often much higher during the winter months than in the summer. Low levels of $\mathrm{CO}$ relative to the levels of oxygen, in inhaled air can be highly toxic and can alter hemoglobin so that it is no longer able to deliver oxygen to organs and tissues. CO disperses quickly in the air, so moderate and high levels of the gas are usually detected only in areas with significant motor vehicle traffic or within enclosed spaces where it may accumulate. The interior $\mathrm{CO}$ concentrations of automobiles driving in numerous cities around the world are measured by the researchers and have considered such variables as road type, traffic conditions, vehicle speed, and time of day. The average vehicle cabin $\mathrm{CO}$ concentrations are ranging from 9.1 to $22.3 \mathrm{ppm}$ (Parts per Million). The researchers compared it to an average ambient air CO level, calculated from measurements at fixed stations near the commuter routes, of between 2.2 and $2.3 \mathrm{ppm}$. Typically the vehicle cabin levels were about seven times higher than those at the remote sites [22].

\subsubsection{Carbon Monoxide (CO) sensors}

When the vehicle with the closed doors is entangled in a traffic jam or in a traffic signal, the CO from the exhaust of other nearby vehicles will be easily pulled in to the vehicle cabin which can create the major disaster to the persons inside the cabin. The proposed system has an embedded system which detects the presence of $\mathrm{CO}$ inside the vehicle cabin. A CO sensor is a device that detects the presence of the $\mathrm{CO}$ gas in order to prevent the $\mathrm{CO}$ poisoning. Figure3 shows the MQ-7 Carbon Monoxide sensor. Sensitive material of MQ-7 gas sensor is $\mathrm{SnO} 2$, with lower conductivity in clean air. It make detection by the method of cycle high and low temperature, and detects $\mathrm{CO}$ when low temperature (heated by $1.5 \mathrm{~V}$ ) and the sensor's conductivity is higher along with the gas concentration rising. When high temperature (heated by $5.0 \mathrm{~V}$ ), it cleans the other gases adsorbed under low temperature. The specifications of the MQ-7 sensor are shown in the table1. A simple electronic circuit is used which converts the change of conductivity to its corresponding output signal of gas concentration. MQ-7 gas sensor has high sensitivity to Carbon Monoxide. The sensor could be used to detect different gases contains CO and is a low cost and suitable for different applications [5].

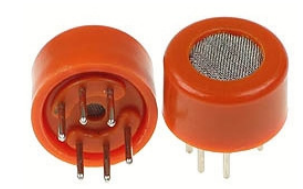

Figure 3: Carbon Monoxide Sensors 


\section{Features:}

- High sensitivity

- Detection Range: 10-1000 ppm of CO

- Response time $:<150 \mathrm{~s}$

- Heater voltage: $5.0 \mathrm{~V}$

- Dimensions: $16 \mathrm{~mm}$ diameter, $10 \mathrm{~mm}$ high excluding pin, pins-6 mm High.

\subsubsection{Oxygen Sensors}

The oxygen gas sensor, sometimes called as electrochemical gas sensors which have properties that make them attractive for long term monitoring application and real time measurement, process, control, and safety applications. The sensor measures the proportion of oxygen $\left(\mathrm{O}_{2}\right)$ in the gas or in ambient air. The oxygen gas sensor has a short response time, high stability and a long life time. Now a day's the oxygen level in the atmosphere get declines and the level of carbon dioxide goes in the increasing order. The most common application is to measure the exhaust gas concentration of oxygen for internal combustion engines in automobiles and other vehicles. They are simple in structure, reliable and rugged in use, modest in cost and low Power consumption. A shortage of oxygen in a cabin while a person is driving a vehicle is thought to be one cause of reducing the degree of concentration of the person and making that person gets sleepy [9].

Further, this system prevents the person from developing high-altitude sickness when a vehicle runs in highlands. The pollutant levels inside the vehicle cabin mostly exceed those in the ambient air or at the roadside. The air pollution accumulating in the interior of automobiles consists almost exclusively of gasoline and diesel exhaust. In confined spaces, the level of oxygen are directly affects the persons inside, and have no open area, that is like travelling in a car while $\mathrm{A} / \mathrm{C}$ is running. The oxygen content of atmosphere is about $21 \%$. Oxygen deprivation also affects the human brain functions, and sometimes leads to induces violent psychotic behavior.

Table.1. The specifications of MQ-7

\begin{tabular}{|c|c|c|c|}
\hline \multicolumn{3}{|c|}{ Model No. } & $M Q-7$ \\
\hline \multicolumn{3}{|c|}{ Sensor Type } & Semiconductor \\
\hline \multicolumn{3}{|c|}{ Standard Encapsulation } & Plastic \\
\hline \multicolumn{3}{|c|}{ Detection Gas } & Carbon Monoxide \\
\hline \multicolumn{3}{|c|}{ Concentration } & 10-10000ppm Co \\
\hline \multirow{4}{*}{ Circuit } & Loop Voltage & $V_{c}$ & $\leq 10 \mathrm{VOC}$ \\
\hline & Heater Voltage & $V_{H}$ & $\begin{array}{l}\text { 5.OV } 00.2 \mathrm{~V} A \text { AOORD (High) } \\
1.5 \mathrm{~V} \pm 0.1 \mathrm{~V} \text { ACorDO (LOW) }\end{array}$ \\
\hline & Heater Time & $T_{\mathbf{L}}$ & $60 \pm 1 \mathrm{~S}$ (High) $90 \pm 1 \mathrm{~S}$ (Low) \\
\hline & $\begin{array}{l}\text { Load } \\
\text { Resistance }\end{array}$ & $\mathrm{R}_{\mathbf{L}}$ & Adjustable \\
\hline \multirow{5}{*}{ Character } & $\begin{array}{c}\text { Heater } \\
\text { Resistance }\end{array}$ & $R_{H}$ & $31 \Omega \pm 3 \Omega$ (Room Tem.) \\
\hline & $\begin{array}{c}\text { Heater } \\
\text { consumption }\end{array}$ & $P_{H}$ & $\leq 350 \mathrm{mV}$ \\
\hline & $\begin{array}{c}\text { Sensing } \\
\text { Resistance }\end{array}$ & $R_{s}$ & $2 \mathrm{~K} \Omega-20 \mathrm{~K} \Omega($ in 100 ppm $\mathrm{CO})$ \\
\hline & Sensitivity & $S$ & Rs(in air)/Rs(100ppm CO) $\geq 5$ \\
\hline & Slope & $a$ & $\leq 0.6\left(\mathrm{R}_{300 \mathrm{ppm} /} / \mathrm{R}_{100_{\mathrm{ppm}}} \mathrm{CO}\right)$ \\
\hline \multirow{3}{*}{ Condition } & Humi & $i t y$ & $20^{\circ} \mathrm{C} \pm 22^{\circ} \mathrm{C} ; 65 \% \pm 5 \% \mathrm{RH}$ \\
\hline & \multicolumn{2}{|c|}{ Standard test circuit } & $\begin{array}{l}\mathrm{V}_{\mathrm{C}}: 5 . \mathrm{OV} \pm \mathrm{O} .1 \mathrm{~V} ; \\
\mathrm{V}_{\mathrm{H}}(\mathrm{High})=5 . \mathrm{OV} \pm 0.1 \mathrm{~V} ; \\
\mathrm{V}_{\mathrm{H}}(\mathrm{LOW}): 1.5 \mathrm{~V} \pm 0.1 \mathrm{~V}\end{array}$ \\
\hline & \multicolumn{2}{|c|}{ Preheat time } & Over 48 hours \\
\hline
\end{tabular}




\subsection{A/D Converter}

The ADC0808 data acquisition component shown in figure5 is a monolithic CMOS device with an 8-bit analog-to-digital converter, 8-channel multiplexer and microprocessor compatible control logic. The 8-bit A/D converter uses successive approximation as the conversion technique. The ADC0808 offers high speed, high accuracy, minimal temperature dependence, excellent longterm accuracy and repeatability, and consumes minimal power. These make the device ideally suitable for application processes and machine control to consumer and automotive applications. The ADC0808M consists of an analog signal multiplexer, an 8-bit successive-approximation converter, and related control and output circuitry.

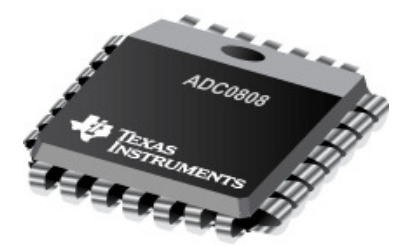

Figure 5: ADC0808

\subsubsection{Multiplexer}

The analog multiplexer selects 1 of 8 single-ended input channels as determined by the address decoder. Address load control loads the address code into the decoder on a low-to-high transition. The output latch is reset by the positive-going edge of the start pulse. Sampling also starts with the positive-going edge of the start pulse and lasts for 32 clock periods. The conversion process may be interrupted by a new start pulse before the end of 64 clock periods. The previous data will be lost if a new start of conversion occurs before the $64^{\text {th }}$ clock pulse. Continuous conversion may be accomplished by connecting the End-of-Conversion output to the start input. If used in this mode an external pulse should be applied after power up to assure start up.

\subsubsection{Converter}

The CMOS threshold detector in the successive-approximation conversion system determines each bit by examining the charge on a series of binary-weighted capacitors (Figure 2). In the first phase of the conversion process, the analog input is sampled by closing switch SC and all ST switches, and by simultaneously charging all the capacitors to the input voltage. In the next phase of the conversion process, all ST and SC switches are opened and the threshold detector begins identifying bits by identifying the charge (voltage) on each capacitor relative to the reference voltage. In the switching sequence, all eight capacitors are examined separately until all 8 bits are identified, and then the charge-convert sequence is repeated.

In the first step of the conversion phase, the threshold detector looks at the first capacitor (weight $=128$ ). Node 128 of this capacitor is switched to the reference voltage, and the equivalent nodes of all the other capacitors on the ladder are switched to REF-. If the voltage at the summing node is greater than the trip-point of the threshold detector (approximately one-half the VCC voltage), a bit is placed in the output register, and the 128-weight capacitor is switched to REF-. If the voltage at the summing node is less than the trip point of the threshold detector, this 128-weight capacitor remains connected to REF+ through the remainder of the capacitor-sampling (bitcounting) process. The process is repeated for the 64-weight capacitor, the 32-weight capacitor, 
and so forth down the line, until all bits are counted. With each step of the capacitor-sampling process, the initial charge is redistributed among the capacitors. The conversion process is successive approximation, but relies on charge redistribution rather than a Successiveapproximation register (and reference DAC) to count and weigh the bits from MSB to LSB.

\subsection{Alarm Circuit}

Safety alarms are electronic alarms designed to alert the user to a specific danger. The hazardous gas like carbon monoxide leaks or its presence is potentially identified by means of various sensors. An audible alarm is usually employed with these sensors to alert people when a dangerous gas has been detected. Sensors are connected to a control unit via low-voltage wiring or a narrow band RF signal which is used to interact with a response device. The proposed system produces alarm whenever the carbon monoxide exceeds the normal level or the oxygen level declines from the required level.

\subsection{Liquid Crystal display}

Liquid Crystal Displays (LCD) have materials, which combine the properties of both liquids and crystals. LCD is a flat electronic visual display. Light modulating properties of liquid crystals are being used for the video display in the LCD. An LCD consists of two glass panels, with the liquid crystal materials sand witched between them LCD are more reliable and energy efficient. Its low power energy consumption makes it to be used in battery powered electronic devices. LCD consists of array of small pixels. Each pixel of an LCD consists of a layer of molecules aligned between two transparent electrodes, and two polarizing filters, the axis of transmission is perpendicular to each other. With no actual liquid crystal between the polarizing filters, light passing through the first filter would be blocked by the second (crossed) polarizer. The Liquid Crystal Display is intrinsically a passive device it is a simple light valve. The managing and control of the data to be displayed is performed by one or more circuits commonly denoted as LCD drivers. The toxic level of carbon monoxide and the oxygen level inside the cabin will be displayed each and every second in the LCD.

\subsection{UART}

UART is a type of asynchronous receiver/transmitter and is an integrated circuit used for serial communications over a computer or peripheral devices serial port. It is usually used to connect to an RS232 interface for modems, printers and similar peripherals. The UART has a feature that, handshake lines for control of an external modem, which is controlled by software. Most of the microcontrollers are included with the UART. For the better coordination of data stream between computer and serial devices, buffering of data are provided by the UARTs. Each UART contains a shift register which performs the conversion of serial and parallel forms. The modem's maximum transmission rate is easily handled by the computer's UART.

\subsection{GSM modem}

GSM (Global System for Mobile Communications: originally from Group Special Mobile) is the most popular standard for mobile telephony systems in the world. The GSM association has its promoting industry trade organization of mobile phone carriers and manufactures, estimate that $80 \%$ of the global mobile market uses this standard. A GSM modem is wireless modem that works with a GSM wireless network. GSM modem requires a SIM card from a wireless carrier in order to operate. The connection between the modem is given in Figure 6. A GSM available service like SMS (Short Message Service) represents alternative modalities to make remote measurements and control. SMS service is suited for remote control applications that requires 
small amount of data and rare activation, like sending alarms, or emergency commands. The system can be interconnected with the car alarm system and alert the owner, on his mobile phone. The system is composed by a microcontroller and a GSM phone.

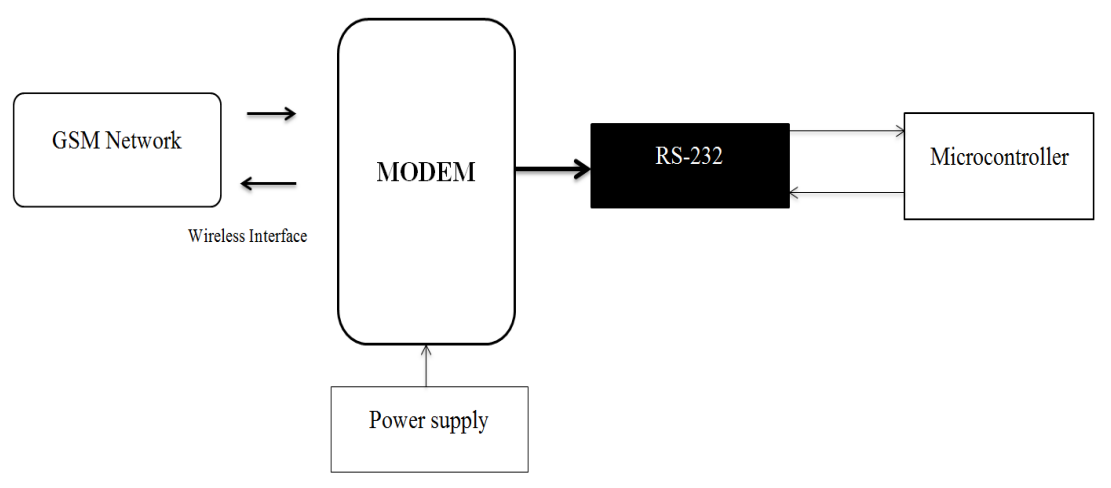

Figure 6: Block diagram of GSM

\section{SOFTWARE DESCRIPTION}

\subsection{Keil Compiler}

The keil C51 C compiler for the 8051 microcontroller is used to solve the complex problems facing embedded software developers. It provides more features than any other $8051 \mathrm{C}$ compiler available today. The microcontroller applications that are written in $\mathrm{C}$ and once complied using the C51 compiler have the efficiency and speed of the assembly language. While starting a new project, first we have to select the microcontroller, that we are going to use for our project from the device database and the $\mu$ Vision IDE sets all compiler, assembler, linker, and memory options. The on-chip peripherals of the microcontroller are accurately simulated by the keil $\mu$ Vision debugger. The hardware configurations can be easily understand by the simulation and also avoids time wasting in setting up of problems. And also we can write and test the applications before the availability of the hardware [18]. The in-System debugger or USB-JTAG adapter can be used to download and test the program code on the target system. The C51 compiler translates the $\mathrm{C}$ source files into re-locatable object modules which contains full symbolic information for debugging with micro vision debugger or an in-circuit emulator. This Keil C51 compiler generates fast compact codes for the 8051and its derivatives. It supports a number of $\mathrm{C}$ language extensions that have been added to support the microcontroller architecture like data types, pointers, memory types, Interrupts.

\subsection{Embedded C}

Embedded $\mathrm{C}$ is not a part of the $\mathrm{C}$ language as such. Rather, it is a $\mathrm{C}$ language that is the subject of a technical report by the ISO working group named "Extensions for the Programming Language C to support Embedded Processors". It aims to provide portability and access to common performance-increasing features of processors used in domain of the DSP and embedded processing. The embedded $\mathrm{C}$ specification for fixed-point, named address spaces and named register gives the programmers direct access to the features in the target processor there by significantly improving the performance of the applications. The hardware I/O extension is a portability feature of Embedded C. Its goal is to allow easy porting of device-driver code between 
systems. Embedded C is designed to bridge the performance mismatch between the Standard C and the embedded hardware and application architecture. It extends the $\mathrm{C}$ language with the primitives that are needed by signal processing applications and that are commonly provided by the DSP processors. Embedded C makes life easier for application programmers. The primitives provided are the primitives that fit the conceptual model of the application which brings back the roots of $\mathrm{C}$ to the embedded systems as primarily a high-level language means of accessing the processor [10].

\subsection{Software Development}

There are several different ways of writing code for embedded systems depending on the complexity of the system and the amount of time and the money that can be spent. Developing software for ready-built hardware is generally easier than for discrete designs. Many ready built designs provide libraries and additional software support which dramatically cut the development time. The traditional method of writing code has centered on a two pronged approach based on the use of microprocessor emulation. The Embedded software development steps are shown in figure 7. The Embedded software is actually written, complied, assembled, linked, debugged and tested at the host system. Then the software is downloaded in to the Atmel 89c51 Microcontroller using the universal programmer.

The universal programmer is connected with the host system through the RS232 serial port communication. The microcontroller is removed from the universal programmer socket and then the microcontroller is placed in to the embedded target system. The Embedded software development and testing were usually done at the host system rather than the target system, since the target system may not have a keyboard, screen, disk drive and the other peripherals necessary for programming. The embedded computing machines make their design more difficult due to complex testing, limited observability and controllability and also with restricted development environments.

\begin{tabular}{|c|c|c|}
\hline HOST SYSTEM & $\begin{array}{l}\text { UNIVERSAL } \\
\text { PROGRAMMER }\end{array}$ & TARGET SYSTEM \\
\hline Debug code & Download & Embedded \\
\hline Compile code & Program & system \\
\hline Debug code & & \\
\hline Test code & & \\
\hline
\end{tabular}

Figure 7: Software Development

\section{IMPLEMENTATION}

The level of the toxic gas CO is continuously sensed by the sensor MQ-7. The level is displayed in the LCD continuously for each and every second. When the level of the toxic gas CO exceeds the normal level of 30ppm or the level of Oxygen decreases the normal level of $19.5 \%$, then the microcontroller proceeds with an alarm. The GSM modem inside the vehicle sends a message to the authorized user about the alarming situation inside the cabin with the levels of the gases monitored by the sensors. Then ventilation is provided, so that the level of the toxic gases can be lowered as early as possible. This provides an immediate response to the situation which is an added advantage of the system. The prototype of the proposed system is shown in Figure8. 


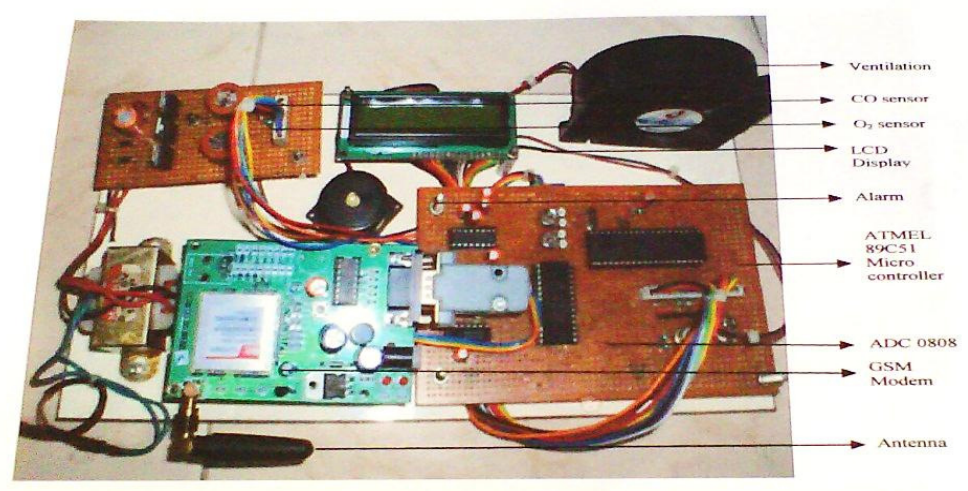

Figure 8: Prototype of the proposed system

\section{CONCLUSION}

An embedded system is designed and tested for toxic gas detection inside a vehicle cabin using ATMEL 89C51 microcontroller. Toxic gas like CO is less sensible by human which endangers the human lives. This critical situation can be avoided by implementing the sensors for sensing the level of CO and oxygen level and is displayed every second. When the Co level exceeds normal level that is $\mathrm{CO}$ is greater than $30 \mathrm{ppm}$ and if the Oxygen level decreases below the normal level of 19ppm then the designed system provides an alarm and also the warning message to the authorized user. Ventilation is immediately provided in the cabin, whenever this critical situation occurs. This prototype can also be used at Home; Educational and working institutions for monitoring the indoor air quality which intern enhance the quality of working environment.

\section{REFERENCES}

[1] A.R.Ali, E. Imran Zualkerman, and Fadi Aloul, "A Mobile GPRS-Sensors Array for Air Pollution Monitoring", vol. 8, pp. 415-422, 2010.

[2] F.-S. Bai, Y.-L. Liu, "Design of Fault Monitoring Alarm System for Networks Based on GSM SMS," pp. 45-67, 2010.

[3] Al-Ali, member, IEEE, Imran Zualkerman, and Fadi Aloul, "A Mobile GPRS-Sensors Array for Air Pollution Monitoring", vol. 6,pp. 410-422, Oct.2010.

[4] Da-Jeng Yao,"A gas sensing system for indoor air quality control and polluted environment monitoring ”, pp. 11-14, 2009.

[5] W. Chung and C. H.Yang, "Remote monitoring system with wireless sensors module for room environment," Sens. Actuators B, vol. 113, no. 1, pp. 35-42, 2009.

[6] F. Tsow, E Forzani, A. Rai, R. Wang, R. Tsui, S. Mastroianni, C. Knobbe, A. J. Gandolfi, and N. J. Tao, "A wearable and wireless sensor system for real-time monitoring of toxic environmental volatile organic compounds,” IEEE Sensors J., vol. 9, pp. 1734-1740, Dec. 2009.

[7] Chen Peijiang, Jiang Xuehua, "Design and implementation of Remote monitoring system based on GSM”, vol. 42, pp. 167-175, 2008.

[8] LIU Zhen-ya, Wang zhen-dong, Chen Rong, "Intelligent Residential Security Alarm and Remote Control System Based on Single Chip Computer", vol. 42, pp.143-166, 2008.

[9] N. Kularatna and B. H. Sudantha, "An environmental air pollution monitoring system based on the IEEE 1451 standard for low cost requirements "IEEE Sensors J., vol. 8, pp. 415-422, Apr. 2008. 
[10] Ben Gaid, M.; Kocik, R.; Sorel, Y.; Hamouche, R. A methodology for improving software design lifecycle in embedded control systems. In Proc. of Design, Automation and Test in Europe (DATE), Munich, Germany, March 2008.

[11] Y. J. Jung, Y. K. Lee, D. G. Lee, K. H. Ryu, and S. Nittel, “Air pollution monitoring system based on geosensor network," in Proc. IEEE Int. Geoscience Remote Sensing Symp., 2008, vol. 3, pp. 13701373.

[12 ]M. Gao, F. Zhang, and J. Tian, "Environmental monitoring system with wireless mesh network based on embedded system", in Proc. 5th IEEE Int. Symp. Embedded Computing, 2008, pp. 174-179.

13] J. W. Kwon, Y. M. Park, S. J. Koo, and H. Kim, "Design of air pollution monitoring system using ZigBee networks for ubiquitous-city," in Proc. Int. Conf. Convergence Information Technology, 2007, pp. 1024-1031.

[14] Ruijie Zhang Funjun He, Zhijiang Du and Lining Sun, “An Intelligent Home Environment Inspecting Robot", vol. 42, pp.140-169, 2007.

[15] H.-M. Tsai, C. Saraydar, T. Talty, M. Ames, A. Macdonald, and O. Tonguz, "Zigbee-based intra-car wireless sensor network," in Proc.IEEE Int. Conf. on Communications (ICC'07), June 2007, pp. 3965-3971.

[16] Ioan, Lita, Ion Bogdan Cioc, Daniel Alexandru Visan, "A New Approach Of automobile Localization System Using GPS and GSM/GPRS Transmission”, vol. 8, pp. 415-422, 2006.

[17] H. W. Huang, The HCS12/9S12: An Introduction to Hardware and Software Interfacing, 1 st ed. Florence, KY: Thomson Delmar Learning, 2006.

[18] M. Petrova, J. Riihijarvi, P. Mahonen, and S. Labella, "Performance study of IEEE 802.15.4 using measurements and simulations," in Proc.IEEE Wireless Commun. and Networking Conf. (WCNC'06), Las Vegas, USA, Apr. 2006, pp. 487-492.

[19] M. AbuJayyab, S. Al Ahdab, M. Taji, Z. Al Hamdani, and F. Aloul, "Pollumap: A pollution mapper for cities," in Proc. IEEE Innovationsin Information Technology Conf., Dubai, UAE, Nov. 2006, pp. $1-5$.

[20] Goran Jurkovic, Mario Iagar, "GSM wireless Solutions in distributed embedded systems designed for automatic control" vol.6, pp. 14-18, 2004.

[21] K. Galatsis, W. Wlodarsla, K. Kalantar-Zadeh and A. Trinchi, "Investigation of gas sensors for vehicle cabin air quality monitoring," vol. 42, pp. 167-175, 2002.

[22] K. Galatsis, W. Wlodarski, Y.x. Li and K. Kalantar-zadeh, "Vehicle cabin air quality monitor using gas sensors for improved safety," pp. 143-164, 2000. 